\title{
Recurrent Solitary Fibrous Tumor of the Falx Cerebri With Intraventricular Extension
}

\author{
-Case Report- \\ Kohsuke Teranishi, Takuji YAmAmoto, Yasuaki NAKAO, Hideo OSADA, \\ Ryo WADA*, and Kentaro MORI \\ Departments of Neurosurgery and ${ }^{*}$ Clinical Pathology, \\ Juntendo University Shizuoka Hospital, Izunokuni, Shizuoka
}

\begin{abstract}
A 61-year-old male presented with a recurrent solitary fibrous tumor (SFT) arising from the falx cerebri with intraventricular extension manifesting as nausea and vomiting. Magnetic resonance imaging showed the heterogeneously enhanced tumor in the falx, which extended to the bilateral lateral ventricles and the third ventricle. Total tumor removal was performed via the bifrontal interhemispheric approach. Histological examination showed mostly spindle cells with rich intercellular fibers. Immunohistochemical examination showed strong staining for CD34 in the cytoplasm but no staining for epithelial membrane antigen. Reexamination of the two previous tumor specimens, previously identified as fibrous meningioma, found SFT. The differential diagnosis of SFT in the central nervous system from fibrous meningioma and hemangiopericytoma requires immunohistochemistry and electron microscopy.
\end{abstract}

Key words: solitary fibrous tumor, fibrous meningioma, hemangiopericytoma

\section{Introduction}

Solitary fibrous tumor (SFT) is generally located in the pleura ${ }^{6)}$ but may also occur in the retroperitoneum, deep soft tissue of the proximal extremities, abdominal cavity, trunk, head, and neck. ${ }^{8,9)}$ SFT is believed to be extremely rare in the central nervous system (CNS). SFT in the CNS shows similar histological characteristics to fibrous meningioma and hemangiopericytoma (HPC). ${ }^{11}$ Many recent reports suggest that most tumors previously identified as HPC are actually SFT. ${ }^{3,4,15)}$ Accurate histological diagnosis depends on both immunohistochemical and electron microscopy examinations.

We describe a case of recurrent SFT of the falx cerebri with intraventricular extension, which was previously identified as fibrous meningioma.

\section{Case Report}

A 61-year-old male was admitted to our hospital complaining of nausea and vomiting. He had a

Received October 3, 2006; Accepted March 26, 2007 history of brain tumor removal twice in our institution. The first operation was performed 10 years ago under a diagnosis of falx meningioma. The histological diagnosis was fibrous meningioma. The second operation was performed for local recurrence 2 years later. A small amount of the tumor was left along the pericallosal arteries at the first and second operations. However, he failed to come to our outpatient clinic for several years.

On admission, he had no neurological deficit. Computed tomography (CT) showed an isodense mass located between the bilateral frontal lobes, attached to the falx cerebri, and associated with severe perifocal edema. Magnetic resonance (MR) imaging showed the tumor as isointense on $\mathrm{T}_{1}$-weighted images, iso- to hyperintense on $\mathrm{T}_{2}$ weighted images, and heterogeneously enhanced by gadolinium-diethylenetriaminepenta-acetic acid. The tumor in the falx extended to the bilateral lateral ventricles and the third ventricle (Fig. 1). Cerebral angiography demonstrated faint tumor staining from the bilateral pericallosal arteries.

Surgery was performed via the frontal interhemispheric approach. The dumbbell-shaped tumor extended into the bilateral lateral ventricles through 

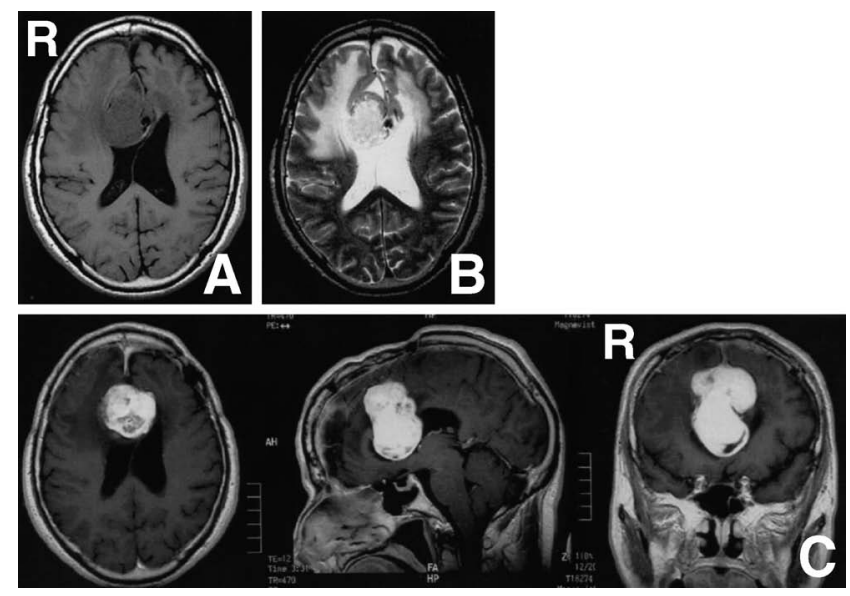

Fig. 1 Magnetic resonance images revealing the mass as isointense on the $T_{1}$-weighted image (A) and iso- to hyperintense on the $T_{2^{-}}$ weighted image (B). $T_{1}$-weighted magnetic resonance images with contrast medium showing the heterogeneously enhanced tumor in the falx extending to the bilateral lateral ventricles and third ventricle through the corpus callosum (C).

a fenestration in the corpus callosum. The grayish pink tumor was elastic hard and tightly attached to the falx cerebri. The cleavage from the normal brain tissue was relatively clear. The intraventricular part of the tumor was elastic soft and easy to dissect from the ependium.

Histological examination showed that most of the tumor cells were spindle cells with clear nuclei and rich eosinophilic intercellular fibers (Fig. 2A). The cellular architecture was partially patternless. Thinwalled blood vessels were present, some of which had the so-called staghorn appearance. Immunohistochemical staining showed strong reactivity for CD34 in the cytoplasm of the tumor cells (Fig. 2B), and positive staining for vimentin (Fig. 2C). Silver staining indicated the rich collagen background, but did not surround individual cells (Fig. 2D). There was no staining for glial fibrillary acidic protein, S-100 protein, epithelial membrane antigen (EMA), or cytokeratin. Ki-67 staining was positive in approximately $5 \%$ of the tumor cells. Electron microscopy showed the tumor cells consisted of spindle cells with irregular elongated cell processes in collagen matrix. Cytoplasm contained dilated rough endoplasmic reticulum, liposomes, mitochondria, and intermediate filaments, but no basal lamina or intercellular junctions (Fig. 3).

The final diagnosis was SFT based on the histological, immunohistochemical, and electron microscopy findings. The tumor specimens obtained at

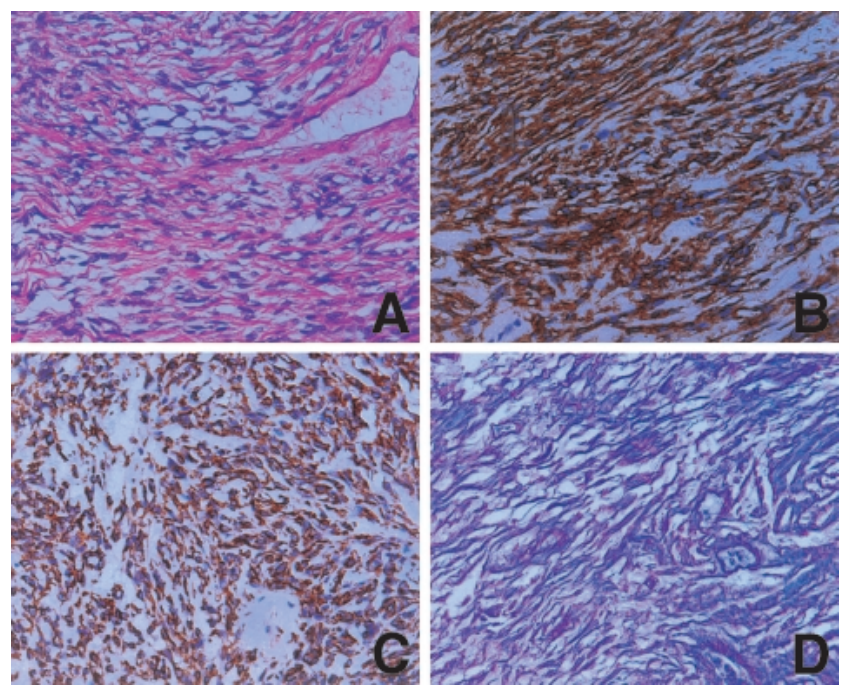

Fig. 2 Photomicrographs of the tumor showing (A) the spindle cells interposed by eosinophilic collagen fibrous bands, and staghorn-type vessels (hematoxylin and eosin stain, $\times$ 200), (B) strong reactivity for $\mathrm{CD3} 4(\times 200)$, (C) strong positivity for vimentin in the tumor cells $(\times 200)$, and $(D)$ rich collagen background (silver stain, $\times 200$ ).

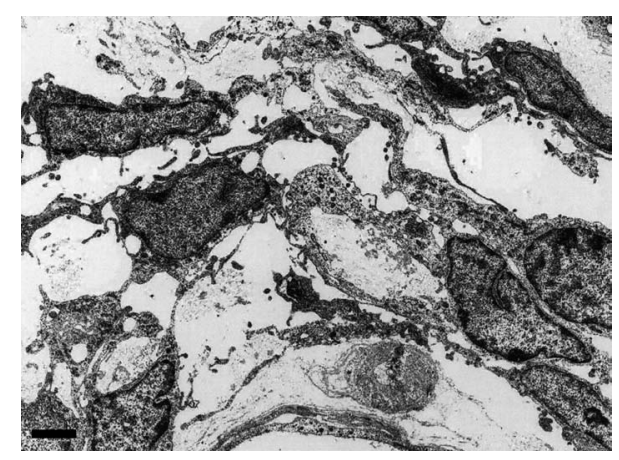

Fig. 3 Electron micrograph of the tumor showing spindle cells with irregular elongated cell processes. The cytoplasm contains dilated rough endoplasmic reticulum, liposomes, mitochondria, and intermediate filaments, but no basal lamina or intercellular junctions. $\quad$ Bar $=\mathbf{5} \mu \mathrm{m}$.

the first and second operations were reexamined. Immunohistochemical examination showed diffuse staining for CD34 and vimentin, and no staining for EMA and cytokeratin. Ki-67 staining was positive in $5-10 \%$ of tumor cells. The reconsidered diagnoses for the previous tumors were both SFT.

The postoperative course was uneventful. The patient was discharged without neurological deficit. 


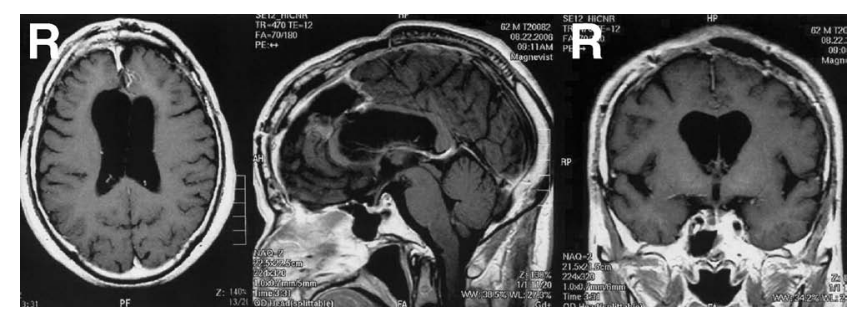

Fig. 4 Postoperative $T_{1}$-weighted magnetic resonance images with contrast medium showing the tumor was completely removed.

Postoperative MR imaging showed that the tumor had been completely removed (Fig. 4).

\section{Discussion}

SFT in the CNS is generally considered to be rare, with only 50 reported cases, ${ }^{1,6-9,11-13,16)} 36$ of meningeal SFT and 14 of spinal SFT.9) However, the number of cases has been increasing since the 1990s. The histological features of SFT show a wide range, from heterogeneous, multinodular, partially sclerotic lesions to monotonous, highly cellular lesions. ${ }^{1,16)}$ Patterns include the so-called patternless pattern, storiform pattern, and neurofibroma-like features. Mitotic figures are rare. ${ }^{4)}$ Immunohistochemical examination shows strong reactivity for $\mathrm{CD} 34,{ }^{2)}$ and may be positive for vimentin, but negative for EMA, S-100 protein, and keratin. Ultrastructural examination shows the distribution of spindle or round cells with rich collagenous bundles between the individual cells, but absence of intercellular junctions and basal lamina. ${ }^{1)}$

The differential diagnosis includes fibrous meningioma, HPC, schwannoma, fibrosarcoma, myofibrosarcoma, and others. ${ }^{4,7}$ Fibrous meningioma includes whole formation or psammoma bodies not seen in SFT. The identification of rich reticulin fibers around SFT cells by silver staining is useful to differentiate from fibrous meningioma. ${ }^{5)}$ Immunohistochemical staining for S-100 protein is positive for schwannoma and fibrous meningioma. ${ }^{1,12)}$ Currently, SFT is difficult to distinguish from HPC by light and electron microscopy. ${ }^{4}$ Ultrastructural examination of "true" HPC shows normal pericytes that possess cellular elements showing elongated cell processes, pericellular basal lamina, plasmalemmal pinocytosis, and varying number of microfilaments. ${ }^{4,12)}$ SFTs also display such features. Immunohistochemical examination shows both SFT and HPC express CD34 and bcl-2 antigen, but are negative for EMA and S-100 protein., ${ }^{4,16)}$ Based on the absence of pericytic differentiation in most HPCs
Table 1 Immunohistochemical features of solitary fibrous tumor, hemangiopericytoma, and fibrous meningiom

\begin{tabular}{lccc}
\hline & $\begin{array}{c}\text { Solitary } \\
\text { fibrous } \\
\text { tumor }\end{array}$ & $\begin{array}{c}\text { Hemangio- } \\
\text { pericytoma }\end{array}$ & $\begin{array}{c}\text { Fibrous } \\
\text { meningioma }\end{array}$ \\
\hline CD34 & ++ & + & + \\
Vimentin & + & + & ++ \\
EMA & - & - & + \\
S-100 protein & - & - & + \\
\hline
\end{tabular}

EMA: epithelial membrane antigen.

and the morphological and immunohistological similarities to SFT, the concept that HPC is a variant of SFT is widely accepted.4) The immunohistochemical features of SFT, HPC, and fibrous meningioma are summarized in Table 1.1,12,15,16)

The 2002 World Health Organization classification of soft tissue tumors acknowledged that most lesions formerly classed as HPC showed no evidence of pericyte differentiation, and are fibroblastic in nature and identical with SFT. ${ }^{3,4)}$ Despite the textbook definition of HPC in the CNS as a distinct tumor entity, lesions previously identified as HPC are probably the cellular type of SFT. The present case of recurrent SFT arising from the falx cerebri with intraventricular extension, which was previously identified as fibrous meningioma, illustrates the changing classification of soft tissue tumors based on the findings of histological, immunohistochemical, and ultrastructural examinations. Understanding of the true biological and clinical nature of SFT in the CNS depends on accurate diagnosis and accumulation of more cases of SFT.

The few reported cases of intracranial SFT have failed to establish the biological behavior, clinical and radiological findings, optimal treatment, and recurrence rate. Intracranial SFTs tend to occur in dura-related areas, such as the convexity, tentorium cerebelli, falx, parasagittal, cerebellopontine angle, fourth ventricle, and so on. ${ }^{12,14)}$ SFTs are generally believed to resemble meningioma clinically and radiologically. ${ }^{14)}$ CT shows SFT as a well circumscribed and partially calcified heterogeneous mass. ${ }^{5,14)}$ MR imaging shows SFT as isointense on $\mathrm{T}_{1}$-weighted images, hyperintense on $\mathrm{T}_{2}$-weighted images, and homogeneous enhancement after intravenous administration of gadolinium. ${ }^{11,17)}$ The clinical behavior of SFT is generally considered to be benign if the tumor is totally removed. ${ }^{12)}$ Recently, stereotactic radiosurgery as adjuvant treatment for residual SFT has been advocated, but experience with more cases is needed.10) 


\section{References}

1) Carneiro SS, Scheithauer BW, Nascimento AG, Hirose T, Davis DH: Solitary fibrous tumor of the meninges: a lesion distinct from fibrous meningioma. A clinicopathologic and immunohistochemical study. Am J Clin Pathol 106: 217-224, 1996

2) Cummings TJ, Burchette JL, McLendon RE: CD34 and dural fibroblasts: the relationship to solitary fibrous tumor and meningioma. Acta Neuropathol (Berl) 102: 349-354, 2001

3) Fletcher CDM: The evolving classification of soft tissue tumours: an update based on the new WHO classification. Histopathology 48: 3-12, 2006

4) Gengler C, Guillou L: Solitary fibrous tumour and haemangiopericytoma: evolution of a concept. Histopathology 48: 63-74, 2006

5) Kim KA, Gonzalez I, McComb JG, Giannotta SL: Unusual presentations of cerebral solitary fibrous tumors: report of four cases. Neurosurgery 54: 1004-1009, 2004

6) Klemperer P, Rabin CB: Primary neoplasms of the pleura. A report of five cases. Arch Pathol 11: 385-412, 1931

7) Kocak A, Cayli SR, Sarac K, Aydin NE: Intraventricular solitary fibrous tumor: an unusual tumor with radiological, ultrastructural, and immunohistochemical evaluation: case report. Neurosurgery 54: 213-217, 2004

8) Macfarlane RG, Galloway M, Plowman PN, Thomas DG: A highly vascular intracranial solitary fibrous tumor treated with radiotherapy and toremifene: case report. Neurosurgery 56: E1378, 2005

9) Miyashita K, Hayashi Y, Fujisawa H, Hasegawa M, Yamashita J: Recurrent intracranial solitary fibrous tumor with cerebrospinal fluid dissemination. J Neurosurg 101: 1045-1048, 2004

10) Nakahara K, Yamada $M$, Shimizu S, Fujii K: Stereotactic radiosurgery as adjuvant treatment for residual solitary fibrous tumor. J Neurosurg 105: 775-776, 2006
11) Nawashiro $H$, Nagakawa $S$, Osada $H$, Katoh $H$, Ohnuki A, Tsuzuki N, Miyazawa T, Shima K, Ogata S, Aida S: Solitary fibrous tumor of the meninges in the posterior cranial fossa: magnetic resonance imaging and histological correlation - case report. Neurol Med Chir (Tokyo) 40: 432-434, 2000

12) Sawauchi $S$, Arakawa H, Taya K, Terao T, Nakazaki $\mathrm{H}$, Numoto RT, Yamaguchi Y, Hashimoto T, Yamaguchi Y, Abe T: [Solitary fibrous tumor of the fourth ventricle: case report]. No Shinkei Geka 31: 551-555, 2003 (Jpn)

13) Shimizu S, Oka H, Kawano N, Utsuki S, Suzuki S, Iwabuchi K, Kan S, Fujii K: Solitary fibrous tumor arising from the falx cerebri-case report. Neurol Med Chir (Tokyo) 40: 650-654, 2000

14) Surendrababu NR, Chacko G, Daniel RT, Chacko AG: Solitary fibrous tumor of the lateral ventricle: CT appearances and pathologic correlation with followup. AJNR Am J Neuroradiol 27: 2135-2136, 2006

15) Suzuki SO, Fukui M, Nishio S, Iwaki T: Clinicopathological features of solitary fibrous tumor of the meninges: An immunohistochemical reappraisal of cases previously diagnosed to be fibrous meningioma or hemangiopericytoma. Pathol Int 50: 808-817, 2000

16) Tihan $T$, Viglione $M$, Rosenblum MK, Olivi A, Burger PC: Solitary fibrous tumors in the central nervous system. A clinicopathologic review of 18 cases and comparison to meningeal hemangiopericytomas. Arch Pathol Lab Med 127: 432-439, 2003

17) Wright $\mathrm{DH}$, Naul LG, Hise JH, Bauserman SC: Intraventricular fibroma: MR and pathologic comparison. AJNR Am J Neuroradiol 14: 491-492, 1993

Address reprint requests to: Kentaro Mori, M.D., Department of Neurosurgery, Juntendo University Shizuoka Hospital, 1129 Nagaoka, Izunokuni, Shizuoka 410-2295, Japan.

e-mail:kmori@med-juntendo.jp 